\title{
IMPACTOS DA AUTONOMAÇÃO EM SISTEMAS PRODUTIVOS: ANÁLISE COM APOIO DA ÁRVORE DA REALIDADE ATUAL
}

\section{AUTONOMATION IMPACTS ON PROODUCTIVE SYSTEMS: ANALYSIS SUPPORTED BY CURRENT REALITY TREE}

\author{
Macáliston Gonçalves da Silva ${ }^{1}$; Miguel Afonso Sellitto ${ }^{2}$ \\ ${ }^{1}$ Universidade do Vale do Rio dos Sinos - UNISINOS - São Leopoldo - Brasil \\ macaliston@ig.com.br \\ ${ }^{2}$ Universidade do Vale do Rio dos Sinos - UNISINOS - São Leopoldo - Brasil \\ sellitto@unisinos.br
}

\section{Resumo}

Este artigo tem como objetivo mapear os impactos da autonomação sobre o sistema produtivo $e$ apresentar, com ajuda de uma Árvore da Realidade Atual (ARA), as relações de causas e efeitos dos procedimentos assumidos. O trabalho está estruturado a partir de revisões da literatura sobre os conceitos e propósitos da autonomação e o Processo de Pensamento da Teoria das Restrições. Após, foi feita uma pesquisa empírica em uma empresa de fabricação eletrônica, na qual foram relatadas as entidades relativas a situação de interesse e construída uma ARA representativa dos resultados encontrados. Dada a formatação e a dinâmica da ferramenta utilizada na pesquisa, seus resultados poderão servir de base para futuros trabalhos e aprofundamentos sobre autonomação.

Palavras-chave: autonomação; jidoka; processos de pensamento (TP); teoria das restrições.

\section{Introdução}

As empresas, para alcançarem vantagens competitivas, devem distinguir-se de seus concorrentes. Para Devaraj et al. (2004), o desempenho do negócio depende do alinhamento dos objetivos da empresa com as capacitações da manufatura. Quanto mais alinhados os objetivos da manufatura com as escolhas de projeto e as respectivas áreas de decisão, maior será o potencial de sucesso e a probabilidade de bom desempenho da manufatura.

Paiva et al. (2004) apontam áreas de decisão em estratégias de manufatura que devem ser consideradas neste alinhamento: (a) capacidade; (b) instalação; (c) equipamentos e processos tecnológicos; (d) recursos humanos; (e) qualidade; (f) integração vertical e relação com fornecedores; (g) sistemas gerenciais; (h) escopo e novos produtos e (i) relação interfuncional.

Os problemas organizacionais de cada empresa devem ser focalizados segundo as 
características e circunstâncias de sua realidade, cabendo salientar a importância de uma análise prévia para o sucesso de implementações estratégicas em manufatura (COONEY, 2002). Em estratégia de manufatura, uma das bases conceituais que pode fornecer alternativas de solução para as citadas áreas de decisão é o Sistema Toyota de Produção (STP). Os resultados que algumas técnicas japonesas surgidas na Toyota ofereceram é um importante contraponto ao modo de pensar a organização da produção nas empresas ocidentais (ZILBOVICIUS, 1999).

Problemas organizacionais ligados à estratégia de manufatura podem ser tratados com auxílio do Processo de Pensamento da Teoria das Restrições (TOC). Esta teoria, proposta por Ely Goldratt, oferece um conjunto de ferramentas que permite identificar, analisar e propor soluções para inúmeras situações (ANTUNES et al., 2004). Dentre as inúmeras referências encontradas na literatura, utilizando as ferramentas do Processo de Pensamento da teoria das restrições, citam-se as pesquisas de Blackstone (2001), Mabin e Balderstone (2003) e Watson et al. (2007), em diversas áreas, incluindo análise e formulação de estratégias.

Os dois pilares de sustentação do STP são: o just-in-time (JIT) e a autonomação (jidoka) (MONDEN, 1984; OHNO, 1997). O objetivo principal deste artigo é explorar o campo da autonomação, com apoio dos processos de pensamento da TOC. A questão central a que se propõe responder é: como pode-se entender os impactos da adoção da autonomação sobre um sistema produtivo?

Os objetivos específicos são: mapear os impactos da aplicação da autonomação sobre os sistemas produtivos e apontar, via ARA (Árvore da Realidade Atual), as relações causa-efeito dos procedimentos assumidos. Dada a formatação da ARA e sua dinâmica, o resultado encontrado poderá servir de base para futuros estudos e aprofundamentos sobre o tema. O método de pesquisa foi o estudo de caso simples. O objeto de estudo foi uma das linhas de produção de uma manufatura do setor eletrônico.

\section{Conceitos da autonomação (jidoka)}

Em 1926, em sua manufatura têxtil, Sakichi Toyoda lança um tear capaz de parar automaticamente quando um dos fios se rompesse ou quando a quantidade programada de tecido fosse atingida. Dessa forma, tornou possível a supervisão simultânea de várias máquinas por um mesmo operador, bastando que este programasse os teares segundo a quantidade previamente estipulada de produto. Buscando o aumento de produtividade a partir da diminuição do número de trabalhadores na fabricação, este conceito foi transferido para a fábrica de automóveis da Toyota, dando origem ao que se conhece hoje por autonomação ou jidoka (GHINATO, 1996; OHNO, 1997). 
Tal inovação ofereceu um contraponto à lógica de um homem por posto de trabalho executando uma tarefa, proposta anteriormente por Taylor. Taiichi Ohno explorou e formalizou as mudanças na Toyota a partir de 1947. A redução da dependência da máquina em relação ao homem é o princípio fundamental deste processo (GHINATO, 1996; ANTUNES et al., 2008).

A palavra jidoka significa apenas automação, sendo ninben no aru jidoka a expressão que dá o verdadeiro significado do conceito e que pode ser interpretada como automação integrada ao ser humano, ou seja, existem dispositivos automáticos, mas a participação humana não é negligenciada no processo. No entanto, a simplificação de uso do termo para jidoka tornou-se usual (MONDEN, 1984). Conforme Monden (1984), autonomação é “automação com a mente humana” ou, segundo Ohno (1997) e Shingo (1996), “automação com um toque humano”. Enfim, máquinas dotadas de procedimento que lembram a inteligência humana (OHNO, 1997).

$\mathrm{Na}$ Toyota, o conceito de autonomação não está restrito às máquinas: também é aplicado às linhas manuais de montagem. Identificada uma anormalidade ao longo da linha, qualquer operador pode parar a produção, desencadeando processos de identificação e eliminação dos problemas.

As paradas, tanto para a linha quanto para máquinas, são sinalizadas por meio de um sistema de informação visual chamado de andon. Andon significa sinal de luz para pedir ajuda. Consiste em um painel luminoso colorido (às vezes acompanhado de sinal sonoro), que indica as condições da linha e aponta o local de solicitação de assistência para todos enxergarem (LIKER, 2005; MONDEN, 1984; OHNO, 1997; SHINGO, 1996).

A autonomação tem como propósitos originais prevenir a geração e propagação de defeitos na produção, tanto para máquinas como em operações manuais, e parar a produção quando foi atingida a quantidade programada. É um mecanismo de controle de anomalias do processo e possibilita a investigação imediata das causas das falhas, pois não permite que a situação que a originou se distancie no tempo (MONDEN, 1984; GHINATO, 1996; OHNO, 1997).

O conceito de autonomação está mais vinculado a autonomia do que a automação. Concede ao operador ou à máquina a autonomia de bloquear o processo sempre que detectar qualquer anormalidade (GHINATO, 1996). A participação da força de trabalho é essencial para a ampliação das oportunidades e manutenção da aplicação da autonomação (GHINATO, 1996; MONDEN, 1984; OHNO, 1997). A autonomação é um processo de "transferência progressiva e contínua do trabalho manual e cerebral para a máquina” (ANTUNES et al., 2008).

\subsection{Propósitos da autonomação}

A máquina automatizada com um toque humano permite liberdade aos operadores para trabalharem simultaneamente com diversas máquinas (multifuncionalidade), reduzindo a demanda 
de operadores na produção e aumentando a eficiência do sistema produtivo (OHNO, 1997). Conforme Antunes et al. (2008), não existe multifuncionalidade sem a aplicação da autonomação. O conceito de autonomação e a multifuncionalidade estão intimamente relacionados e diretamente envolvidos com a eficiência e flexibilidade da força de trabalho. Impactam positivamente na redução de custo de fabricação. Porém, é da flexibilização (multifuncionalidade) que resulta a redução de mão-de-obra (GHINATO, 1996). A autonomação e a multifuncionalidade tornaram possível a adoção de lay-out celular em processos de fabricação.

Duas modalidades de multifuncionalidade podem ser trabalhadas: (a) o sistema de operação de múltiplas máquinas; e o (b) sistema de operação de múltiplos processos. Neste último, o operador trabalha em diversas máquinas de acordo com o fluxo de fabricação. O uso de operações múltiplos processos devem ser preferidos em função dos maiores benefícios conquistados, como a melhora do fluxo dos processos e a elevação da produtividade do trabalhador (GHINATO, 1996; SHINGO, 1996).

A autonomação no STP está associada à eliminação da superprodução e de produtos defeituosos, dois desperdícios significativos e que são observados na manufatura. A superprodução quantitativa é eliminada a partir dos controles de quantidades planejadas que evita o excesso de produção. Já a eliminação de produtos defeituosos na autonomação é combatida com a interrupção do processamento em casos de anormalidades detectadas. Assim, a autonomação colaborado com o desafio de eliminação total de perdas no processo produtivo (OHNO, 1997).

Conforme Ghinato (1996), também, pode-se citar a eliminação secundária da perda por estoque e perda por espera com a aplicação da autonomação. A primeira está relacionada com a eliminação da perda por superprodução quantitativa citada acima e a segunda é tratada pela função controle incorporada ao processamento, liberando o operador para trabalhos efetivos ao longo da produção, ou seja, valorizando a eficiência da mão-de-obra. A autonomação também apresenta a vantagem de poder evitar a participação direta dos trabalhadores em operações de risco à integridade física dos mesmos.

Com multifuncionalidade e sem autonomação, a intensificação do trabalho pode conduzir a um ambiente nocivo, aumentando o risco de acidente e de estafa (GHINATO, 1996). Segundo a lógica 5MQS (Management, Method, Material, Man, Machine, Quality e Safety), perdas relacionadas à segurança (safety) estão diretamente associadas a acidentes de trabalho e afastamento do trabalhador.

Separar, ao máximo, fisicamente o homem da máquina é uma das possibilidades da autonomação, na medida em que isso for implementado, tende a diminuir a possibilidade de ocorrência de acidentes de trabalho durante a operação. A utilização de poka-yoke (dispositivo à prova de falhas) deve ser considerada, também, para garantir a segurança industrial em diversas 
situações, sempre trabalhando com a noção de acidente zero. As perdas relacionadas à segurança tendem a afetar a produtividade, custos com os atendimentos dos acidentados e a moral dos trabalhadores, e podem ser consideradas como perdas sociais (ANTUNES et al., 2008; PASSOS JÚNIOR, 2004).

Mecanismos de detecção de problemas e interrupção do processamento podem ser aplicados, conforme conceitos da autonomação, para a eliminação das quebras de máquinas, também. A Manutenção Produtiva Total - MPT (Total Productive Maintenance - TPM) é um dos elementos que podem auxiliar no alcance e sustentação da quebra zero, maximizando a efetividade dos equipamentos no sistema produtivo (ANTUNES et al., 2008; GHINATO, 1996; SHINGO, 1996). A TPM pode ser útil em objetivos de manutenção, tais como: aumento de confiabilidade e de manutenibilidade dos equipamentos (RIBEIRO e FOGLIATTO, 2009).

Sistemas autônomos podem contribuir, também, para a redução de desperdícios energéticos, desvinculando a atuação das pessoas em funções como, por exemplo, ligar e desligar a alimentação de equipamentos quando estão inoperantes ou a construção de ambientes autonomatizados que colaboram com os objetivos propostos (PASSOS JÚNIOR, 2004).

Dentro do contexto do STP, a combinação entre o CQZD (Controle de Qualidade Zero Defeitos) e a autonomação garante a qualidade do produto ao longo do processo produtivo e efetiva a qualidade assegurada (GHINATO, 1996; ANTUNES et al., 2008). Conforme Shingo (1986), os quatro pontos de sustentação do CQZD são: (i) utilização da inspeção na fonte, assim, tendo a função controle aplicada na origem dos defeitos (causa) e não sobre os resultados (efeito); (ii) utilização de inspeção 100\%; (iii) redução da diferença entre o tempo de detecção de uma anormalidade e o tempo de aplicação da ação corretiva e (iv) reconhecimento do potencial de falha dos trabalhadores, contendo a aplicação de dispositivos à prova de falhas (poka-yoke) para atender a função de controle junto com a função de execução.

Garantir a capacidade de produzir sistematicamente produtos sem defeitos é o objetivo do CQZD (GHINATO, 1996). Ghinato (1996) cita que "quando se diz que o CQZD idealizado por Shingo dá ênfase à questão operacional, não implica que os aspectos motivacionais sejam negligenciados". Conforme Shingo (1996), "para total eliminação dos defeitos, deve-se adotar a inspeção $100 \%$ [...] inspeção por amostragem não é suficiente”.

Ainda segundo Ghinato (1996), o CQZD com a aplicação de poka-yoke em regime de inspeção $100 \%$ é parte essencial na operacionalização da autonomação (função controle). A Figura 1, proposta por Shingo (1986), apresenta o mecanismo da função controle (inspeção na fonte). Ocorrendo em um ciclo curto, conforme citado acima, concentrando-se sobre a causa dos defeitos. 
Figura 1 - Mecanismo da função controle (inspeção na fonte)

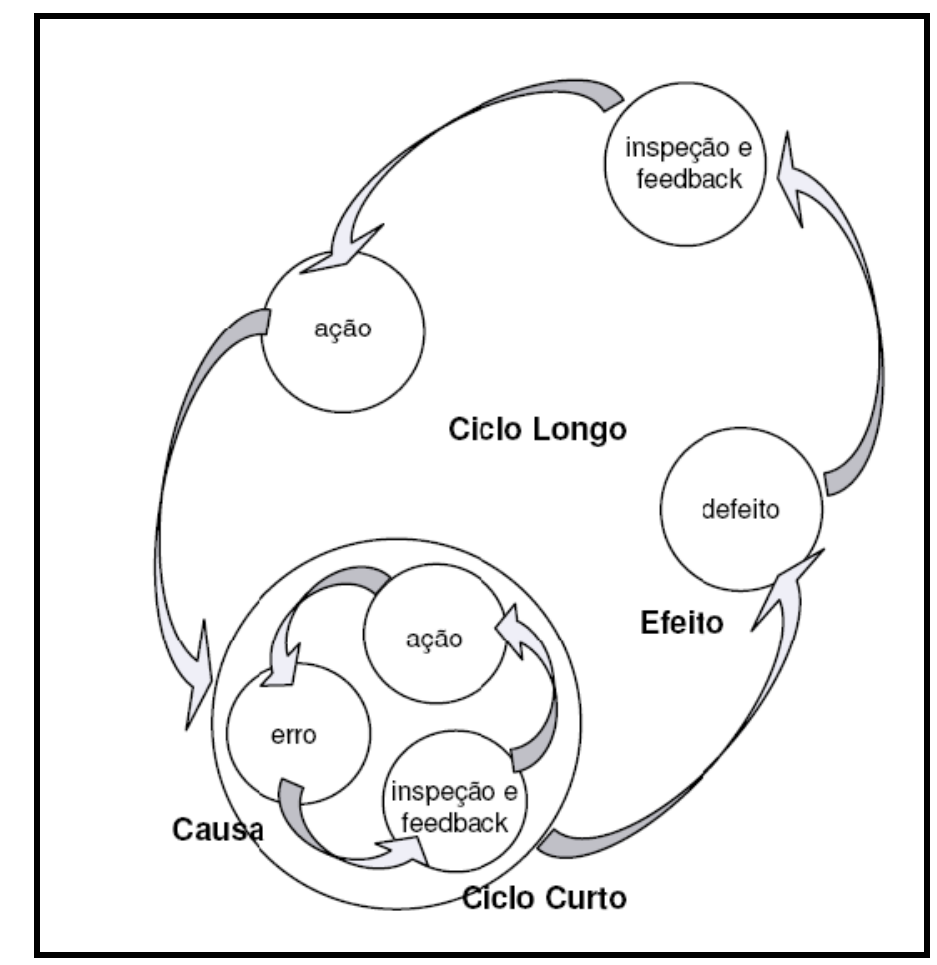

Fonte: Shingo (1986)

O procedimento chave para a eliminação dos defeitos nos produtos concentra-se na imediata pesquisa de levantamento e correção das causas quando da paralisação de uma máquina ou da linha (WOMACK et al., 1992; MONDEN, 1984). Conforme Ohno (1997), parar "para desenvolver uma linha que seja forte e raramente necessite ser parada [...] não há razão alguma para se temer uma parada na linha [...] uma linha de produção que não pára pode ser tanto uma linha perfeita como também uma linha com muitos problemas".

De forma sintetizada, para Monden (1984), a autonomação tem os seguintes propósitos (Figura 2): (a) a redução de custo através da redução da força de trabalho, (b) flexibilidade na produção para alterações na demanda, (c) qualidade assegurada e (d) aumento do respeito à condição humana. Ao fim do processo, resultam quatro objetivos que podem contribuir para as áreas de decisão da estratégia de manufatura, já mencionadas: capacidade de adaptação da produção; redução de custo; melhoria da qualidade; e crescimento do ser humano. 
Figura 2 - Visão de Monden sobre os propósitos da autonomação

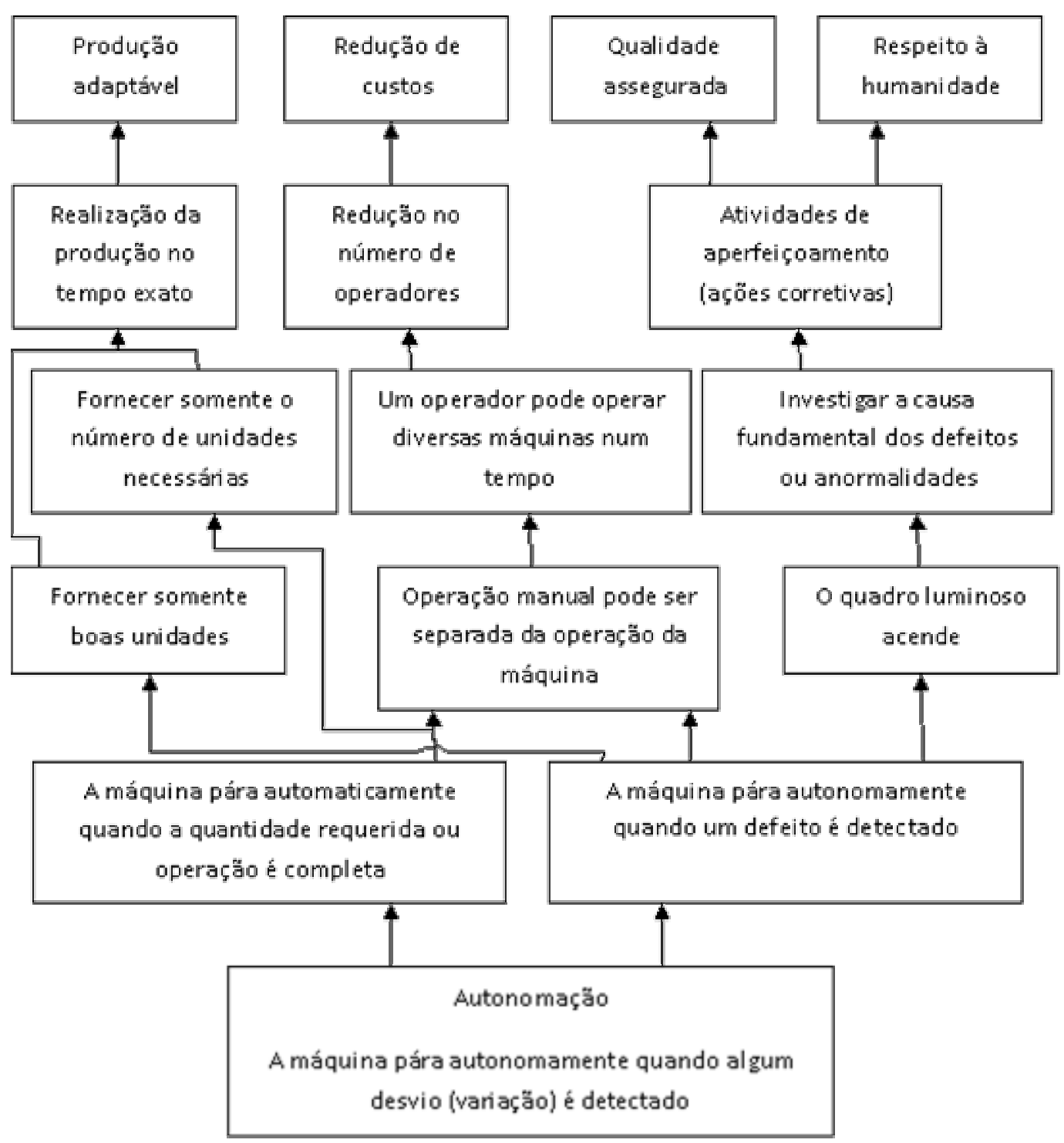

Fonte: Monden (1984)

\section{Processo de pensamento da teoria das restrições}

Na década de 1970, Eliyahu Goldratt, desenvolveu sua Teoria das Restrições (TOC - Theory of Constraints), uma filosofia de melhoramento contínuo empresarial. Seu ponto de partida foram problemas logísticos de produção, criando um método original de administração da produção. Seu trabalho foi disseminado, na década de 1980, com o lançamento do livro A Meta (The Goal), com co-autoria de Jeff Cox.

Na década de 1990, Goldratt, explicita as ferramentas de raciocínio lógico que utilizava de forma intuitiva com o lançamento das obras What Is This Thing Called Theory of Constraints and How Should It Be Implemented? e Mais Que Sorte - Um Processo de Raciocínio (It's Not Luck), cunhando o nome de Processo de Pensamento (Thinking Process - TP) (CORBETT, 1997; COX e 
SPENCER, 1998; MABIN e BALDERSTONE, 2003; RODRIGUES, 1990; WATSON et al., 2007).

A TOC é composta por três campos inter-relacionados (Figura 3): (i) logística; (ii) indicadores de desempenho; e (iii) Processo de Pensamento (CORBETT, 1997; COX E SPENCER, 1998; INMAN et al., 2009; MABIN e BALDERSTONE, 2003; MEHRA et al., 2005).

Figura 3 - Representação dos elementos da Teoria das Restrições

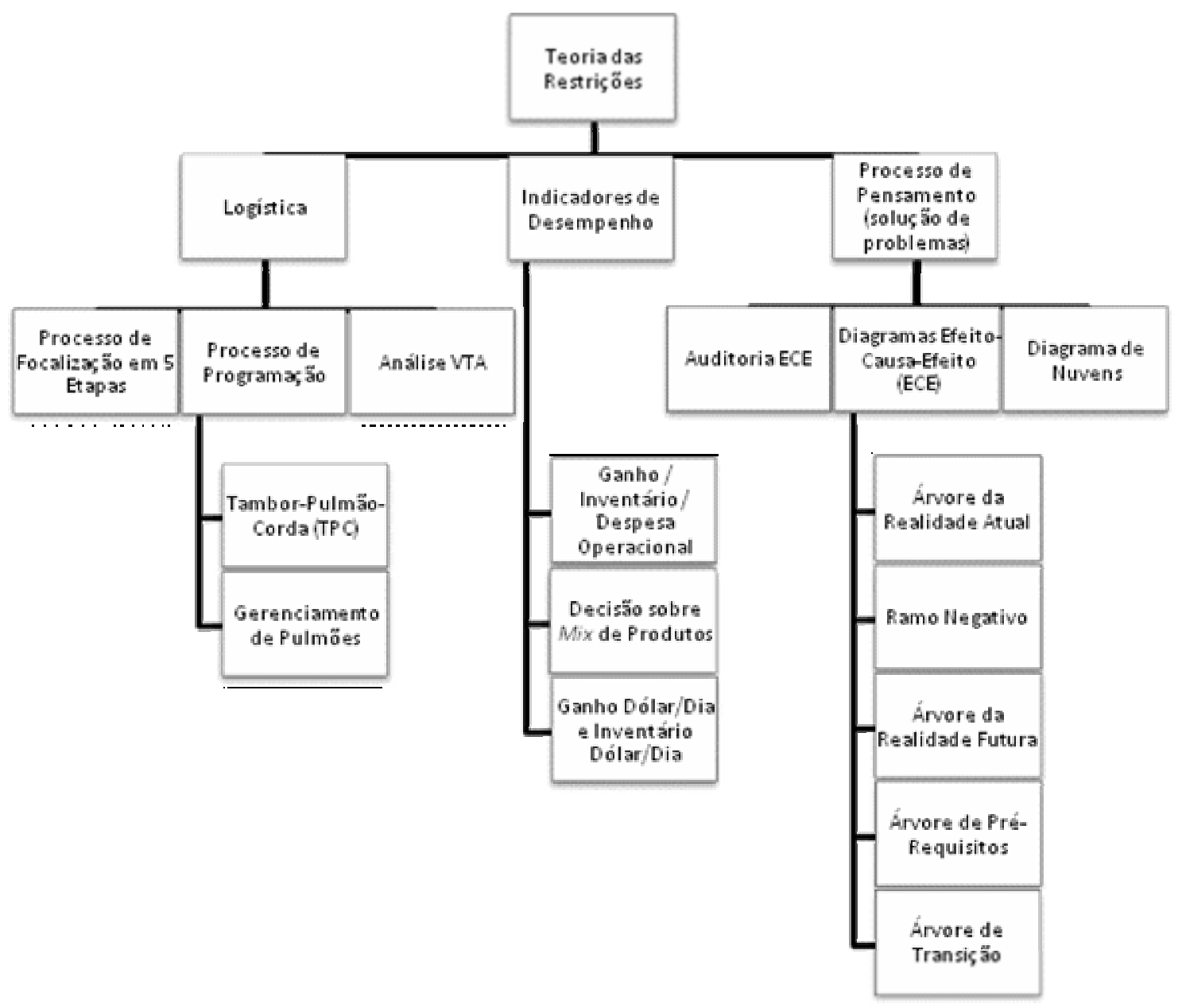

Fonte: Adaptado de Cox e Spencer (1998)

Para Cox e Spencer (1998) e Corbett (1997), o Processo de Pensamento da Teoria das Restrições deve ser visto como um conjunto de ferramentas que podem ser utilizadas individualmente ou ligadas logicamente, sob condições de um relacionamento causal.

Dado o fato de ter ultrapassado os limites da administração da produção, suas ferramentas também são utilizadas em outras áreas do conhecimento empresarial. São diagramas lógicos que auxiliam na explicitação de nossas intuições.

Segundo Blackstone (2001), Mabin e Balderstone (2003), as ferramentas estão sendo aplicadas em vários contextos, como: manufatura, serviços, administrativo, pessoal ou profissional. 
Como pressuposto para o Processo de Pensamento, utilizado por Goldratt, está o raciocínio de que em qualquer sistema, poucas causas explicam os seus muitos sintomas (CORBETT, 1997).

As ferramentas são aplicáveis para a identificação, testes de soluções e superação de problemas organizacionais ou de outra natureza (COX e SPENCER, 1998; KIM et al., 2008). Para Mabin e Balderstone (2003), se combinada a intuição e o conhecimento dos envolvidos na busca da solução ganha-ganha (win-win) para um problema, o Processo de Pensamento com suas ferramentas pode ser aplicado em qualquer tipo de situação. Watson et al. (2007), em seus achados de pesquisa, relatam a existência de várias experiências com o uso das ferramentas do Processo de Pensamento para análise e formulação de estratégias.

Para Goldratt (2004), é essencial para a função dos tomadores de decisão em uma empresa a determinação de: (i) O quê mudar? (ii) Para o que mudar? (iii) Como causar a mudança? E o Processo de Pensamento está estrutura de forma a apresentar um método para responder tais perguntas, utilizando as seguintes ferramentas: (a) Árvore da Realidade Atual (Current Reality Tree - CRT), (b) Evaporação das Nuvens (Evaporating Clouds - EC), (c) Árvore da Realidade Futura (Future Reality Tree - FRT), (d) Árvore de Pré-Requisitos (Prerequisite Tree-PRT) e (e) Árvore de Transição (Transition Tree - TT) (COX e SPENCER, 1998; SCHEINKOPF, 1999; KIM et al., 2008). As relações entre as perguntas, ferramentas e seus efeitos são apresentados no Quadro 1.

Quadro 1 - As ferramentas do processo de pensamento e suas funções.

\begin{tabular}{ccc}
\hline Perguntas & Efeitos & Ferramentas \\
\hline O quê mudar? & $\begin{array}{c}\text { Identificar problemas } \\
\text { centrais }\end{array}$ & Árvore da Realidade Atual \\
\hline Para o que mudar? & Desenvolver soluções & Evaporação das Nuvens \\
\cline { 3 - 3 } & & Árvore da Realidade Futura \\
\hline Como causar a mudança? & Implementar soluções & Árvore de Pré-Requisitos \\
\cline { 2 - 3 } & & Árvore de Transição \\
\hline
\end{tabular}

Fonte: Adaptado de Rahman (2002).

\section{1 Árvore da realidade atual - ARA}

A ARA possibilita aprofundar o conhecimento de uma organização e seu ambiente, identificando os problemas centrais (core problems), descritos por conexões causais a partir dos efeitos indesejados. É uma ferramenta baseada na lógica de causa e efeito que comunica os relacionamentos causais existentes em uma determinada situação de interesse (COX e SPENCER, 1998). 
Antunes et al. (2004) corroboram com a definição do objetivo da ARA, colocando-a como alternativa para o estabelecimento sistemático dos principais problemas de uma determinada situação. Doggett (2005) concluiu que o desenvolvimento da ARA orienta a verbalização da intuição de forma lógica, conduzindo à identificação de um problema central.

O estudo de Kim et al. (2008) explicita que a ARA é uma ferramenta eficaz para questões políticas e estratégicas, ou seja, restrições não necessariamente físicas. Neste sentido, atuando diretamente sobre o fator o quê mudar?

Boyd et al. (2001) e Gupta et al. (2004) compartilham desta posição e propõem em seus trabalhos uma abordagem para formulação de estratégias através das ferramentas do Processo de Pensamento. Assim como Klein e Debruine (1995), que identificaram deficiências nas estratégias de empresas e até setores industriais, através da aplicação destes métodos.

Segundo Cox e Spencer (1998) e Goldratt (2004), para a construção da ARA deve-se seguir, de forma resumida, os seguintes passos: (i) liste os principais problemas observados (ou EIs efeitos indesejáveis) na situação; (ii) teste a clareza de cada EI; (iii) procure relações causais entre os Eis; (iv) determine quem é causa e quem é efeito; (v) continue as ligações entre os EIs valendose da lógica SE-ENTÃO até concluir todas as conexões; e (vi) após expandir a árvore, até o necessário, revisar o trabalho procurando e corrigindo inconsistências.

A ARA é formulada no sentido top-down, partindo dos efeitos para as causas, porém, deve ser interpretada no sentido inverso, ou seja, bottom-top e utilizando a relação SE-ENTÃO (BLACKSTONE, 2001; GUPTA et al., 2004).

Isso é corroborado por Alvarez (1996), citando que a interpretação da ARA deve ser feita lendo-se, sempre, a partir da causa e seguindo a lógica: SE ocorrer a 'causa', ENTÃO o 'feito' se manifestará. Os EIs sem entradas podem ser classificados como causas raiz e problemas centrais, ou seja, aqueles que levam ao maior número de efeitos indesejados (ALVAREZ, 1996).

Conforme Cox e Spencer (1998) e Klein e Debruine (1995), a diagramação de causalidade resultante do processo de construção da ARA permite:

a) Identificar o impacto de políticas, procedimentos e ações em uma organização;

b) Comunicar de forma clara e concisa a causalidade das políticas, procedimentos e ações;

c) Identificar o problema central em uma situação;

d) Formar equipes coesas e focalizadas para a eliminação do problema;

Para Goldratt (2004), a base do método do Processo de Pensamento está na construção da ARA, que foi a ferramenta adotada nesta pesquisa. 


\section{A pesquisa}

Dadas as características da Árvore da Realidade Atual (ARA) apontadas pela literatura citada acima, optou-se por sua utilização como forma de representação final dos objetivos propostos por esta pesquisa.

A questão central foi: como pode-se entender os impactos da adoção da autonomação sobre um sistema produtivo? Os objetivos específicos foram: (a) mapear os impactos da aplicação da autonomação sobre um sistema produtivo; e (b) descrever, via ARA, as relações de causa e efeitos dos procedimentos assumidos. Como objeto de estudo, foi escolhida uma linha de produção de uma empresa brasileira de alta tecnologia da indústria eletrônica.

A opção de escolha se dá em função da empresa ser a líder de mercado nacional em seu segmento, trabalhando com o desenvolvimento e fabricação de produtos com tecnologia agregada. Inserida em um mercado (interno e externo) altamente competitivo e reconhecido pela inovação, concorrendo com players internacionais e sob a fiscalização de órgãos reguladores.

A partir das definições e trabalhos, principalmente, de autores como Monden (1984), Ohno (1997), Shingo (1986, 1996), Womack et al. (1992), Ghinato (1996), Liker (2005) e Antunes et al. (2008) foi construída uma base teórica para sustentação dos impactos da autonomação sobre o sistema produtivo examinado.

A primeira parte da pesquisa incluiu um levantamento de campo, no qual os pesquisadores entrevistaram os supervisores de campo e os inspetores de qualidade da linha em estudo. A técnica empregada foi a entrevista semi-estruturada. Algumas perguntas iniciais sobre o que acontece na linha de montagem foram feitas e à medida que as respostas eram dadas, a entrevista era redirecionada pelos pesquisadores, explorando mais os aspectos mais destacados pelos respondentes. A partir dos relatos obtidos, foram listados e consolidados os efeitos indesejados (entidades) mais importantes e mais repetidamente observados na situação de interesse:

(1) Processo produtivo excessivamente dependente de mão-de-obra;

(2) Não há garantia da parada da produção quando atingido volume planejado;

(3) Controle de volume de produção depende exclusivamente do operador;

(4) Cada operador preocupa-se apenas com seu posto de trabalho (política de ótimos locais);

(5) Surgem estoques intermediários excessivos em alguns pontos e faltas em outros (produção não sincronizada);

(6) Não há garantia da parada da produção quando detectado defeito (depende de operador);

(7) Não há garantia de que defeitos serão detectados (não se utilizam sistemas poka-yoke);

(8) Não há aplicação de inspeção 100\% (CQZD); 
(9) Há expressiva geração de produtos defeituosos;

(10) Sem incentivo de ações imediatas (autonomia);

(11) Poucas ações de melhorias;

(12) Problemas de qualidade;

(13) Operador demasiadamente próximo da máquina;

(14) Baixa flexibilidade da mão-de-obra;

(15) Local de trabalho insalubre e perigoso;

(16) Aumento de acidentes de trabalho e afastamento do trabalhador;

(17) Perdas energéticas;

(18) Excesso de quebras não-planejadas de máquinas;

(19) Máquinas comprometidas;

(20) Mau aproveitamento dos recursos;

(21) Baixa adaptação da produção à demanda;

(22) Aumento de custos do sistema produtivo;

(23) Baixa moral dos trabalhadores;

(24) Falta de respeito à condição humana.

$\mathrm{Na}$ segunda parte, os pesquisadores organizaram os efeitos indesejáveis relatados em forma de ARA (Figura 4).

Conforme a ARA, não aplicar os conceitos da autonomação ou possuir um processo produtivo excessivamente dependente de mão-de-obra (1) e sem garantia de detecção de defeitos (7), significa perda de garantia da parada da produção em casos de detecção de falhas (6) e não aplicação de inspeção 100\% (CQZD) (8), respectivamente. Como efeito, expressiva geração de produtos defeituosos (9). Reforçado, pela falta de incentivo para ações imediatas (autonomia) (10), neste processo produtivo sem a autonomação, que inibe vastas ações de melhorias (11) e provoca o baixo moral dos trabalhadores (23), registram-se problemas de qualidade (12). Ou seja, não há condições adequadas para garantia da qualidade. Sem a autonomação, mantêm-se os operadores fisicamente mais próximos das máquinas (13). Considerando um local de trabalho com riscos para os trabalhadores (15) e a não aplicação de sistemas poka-yoke (7), a combinação destes três fatores (13-15-7) tem como efeito o aumento da probabilidade de acidentes de trabalho e/ou afastamento dos mesmos (16). Isso (16) ou a baixa moral dos trabalhadores (23) contribuem para o desrespeito à condição humana (24). Ainda sem a autonomação, perde-se a garantia da parada da produção quando atingido o volume planejado (2), se combinado com uma política de ótimos locais (4) e o controle de volume de produção é exclusivamente dependente do operador (3), tem-se como conseqüência uma produção não sincronizada (5). Se não há autonomação no processo produtivo (1) ou não há aplicação de sistemas poka-yoke (7), então, existem perdas energéticas (17). Assim 
como, se combinados (1- 7) com o excesso de quebras não-planejadas de máquinas (18), tem-se máquinas comprometidas (19). Máquinas comprometidas (19), perdas energéticas (17) ou a baixa flexibilidade da mão-de-obra (14) refletem em mau aproveitamento dos recursos (20). A combinação deste efeito (20), com problemas de qualidade (12) e uma produção não sincronizada (5), resulta em baixa adaptação da produção às variações na demanda (21).

Figura 4 - ARA dos impactos da autonomação sobre o sistema produtivo

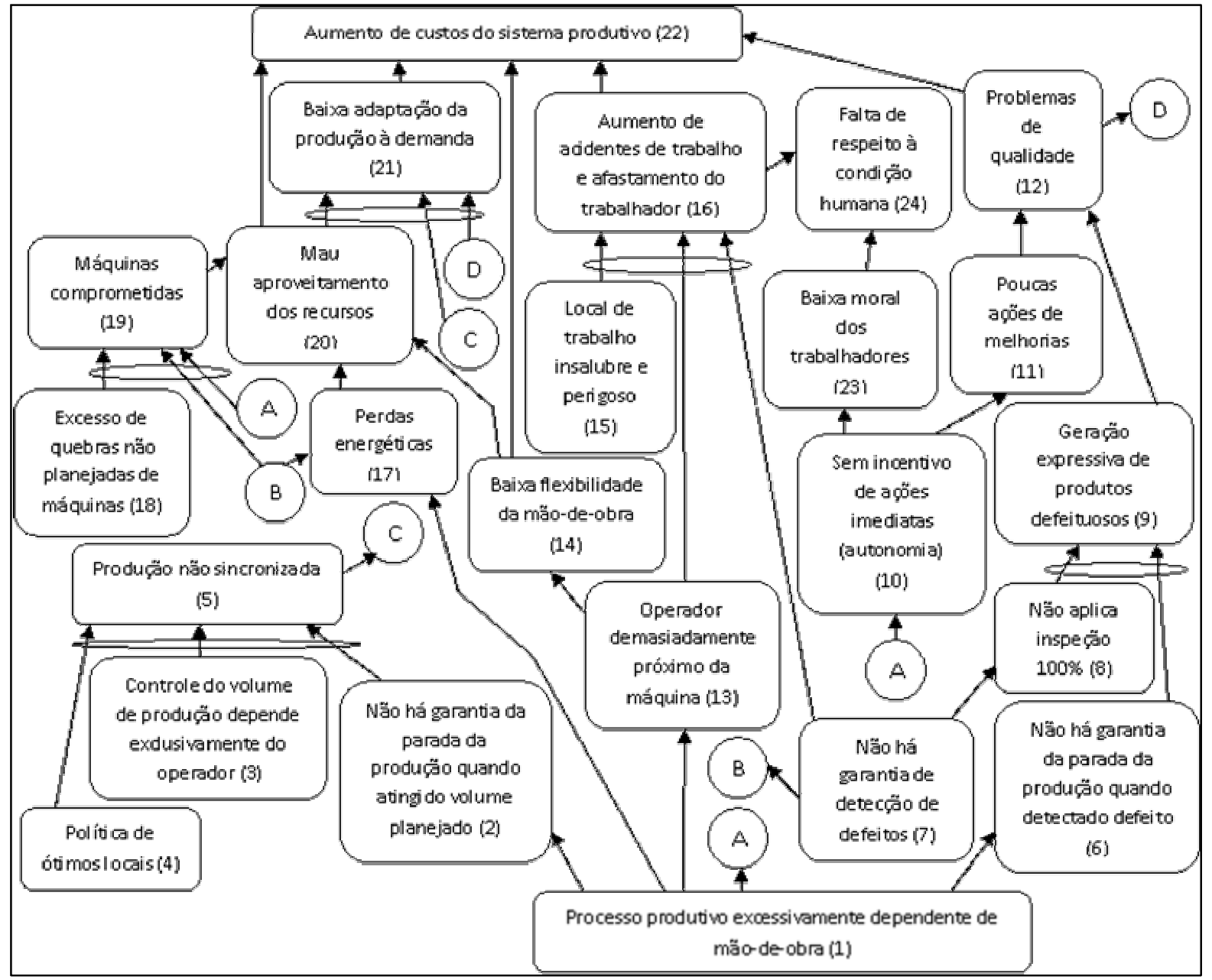

Assim, se há problemas de qualidade (12) ou baixa adaptação da produção à demanda (21) ou mau aproveitamento dos recursos (20) ou aumento de acidentes de trabalho e afastamento do trabalhador (16) ou baixa flexibilidade da mão-de-obra (14), então, há aumento de custos no sistema produtivo (22).

\section{Discussão}

Com a diagramação de causalidades a partir da construção da ARA, foi possível identificar as relações entre os efeitos indesejados da situação em análise. Originados, principalmente, pelo 
processo produtivo excessivamente dependente de mão-de-obra (1) e a falta de garantia de que defeitos serão detectados (não utilização de sistemas poka-yoke) (7), enxergam-se os impactos das políticas adotadas e os conseqüentes resultados destes procedimentos.

A redução de custos do sistema produtivo parece ser o maior ganho com a adoção de políticas centradas nos conceitos da autonomação. De acordo com a ARA, isso é sustentado por um melhor aproveitamento dos recursos internos disponíveis, maior potencial de adaptação da produção às necessidades do mercado, redução de perdas com acidentes de trabalho e redução de perdas com problemas de qualidade na linha de produção.

Outro fator que merece destaque, segundo a representação da ARA, é o impacto positivo que a autonomação pode proporcionar sobre a qualidade no processo produtivo. A partir da adoção de sistemas poka-yoke e paradas automáticas da produção quando há detecção de problemas, pode-se tratar preventivamente a geração e propagação de defeitos ao longo da produção.

Reforçado por um maior grau de liberdade disponibilizado ao operador, que pode refletir em iniciativas de ações de melhorias no trabalho, potencializa-se a atuação imediata dos responsáveis pelo processo para correção de problemas encontrados. Como conseqüência, existe uma forte tendência no atendimento das especificações de montagem, propiciando o alinhamento entre o projetado pela engenharia e o executado pela operação. Também, reduzir defeitos e retrabalhos pode significar um aumento da confiabilidade e conformidade do produto.

Visualizando a ARA, nota-se que a flexibilidade do sistema é impactada pela autonomação, a partir, principalmente, de uma mão-de-obra menos consumida por funções essencialmente ligadas à máquina e um controle mais rígido sobre a eficácia da execução da programação da produção, cria-se a possibilidade da multifuncionalidade dos operadores. Assim, a flexibilidade conquistada com a mão-de-obra favorece as condições de adaptação da produção a variações de demanda, principalmente as do tipo volume. Esta possibilidade de adequação às condições externas, oferecendo alternativas para responder às mudanças pode representar um diferencial perante a concorrência.

Observa-se que, melhores condições de trabalho e mais autonomia aos operadores podem reforçar os benefícios citados anteriormente, dado a tendência de participação mais efetiva da força de trabalho sobre as iniciativas adotadas em um processo produtivo com estas características. Importante salientar que as colocações anteriores corroboram com os trabalhos de autores como Monden (1984), Ohno (1997), Shingo (1986, 1996), Womack et al. (1992), Ghinato (1996), Liker (2005) e Antunes et al. (2008), que estudaram a autonomação e difundiram seus conceitos.

Por fim, a opção por um sistema produtivo capacitado com os conceitos da autonomação caracteriza-se por decisões relacionadas a equipamentos e processos tecnológicos, políticas de recursos humanos, qualidade e sistemas gerenciais. Entende-se que, a manufatura assumindo a 
autonomação como estratégia, pode estar contribuindo para um melhor desempenho da empresa quando o negócio exige competitividade da produção em custo. Mas, qualidade e flexibilidade, também podem ser consideradas como diferenciais competitivos que podem ser conquistados pela empresa a partir da autonomação.

\section{Considerações finais}

Conforme os objetivos desse trabalho, mapear os impactos da autonomação sobre os sistemas produtivos e demonstrá-los de forma clara, conclui-se que, com a aplicação da Árvore da Realidade Atual (ARA), atendeu-se as expectativas.

A autonomação como estratégia e política de atuação em sistemas produtivos pode ser analisada agora, também, com auxílio de uma representação lógica causa-efeito, como apresentada nesse artigo. Não se pode negar seu potencial de persuasão, principalmente, se acrescentado outras ferramentas do Processo de Pensamento na continuidade de exploração do tema.

A abordagem utilizada nesse trabalho parece pertinente. Aplicar uma das ferramentas do Processo de Pensamento para avançar sobre o assunto em questão, também, pode contribuir para testar o potencial que o método proposto por Goldratt tem a oferecer, conforme lacunas levantadas por Mabin e Balderstone (2003).

Segundo Scheinkopf (1999), o Processo de Pensamento pode ter como objetivos de uso: (i) a comunicação, (ii) a tomada de decisão, (iii) resolução de conflitos, (iv) aprendizagem, (v) desenvolvimento e implementação de políticas e (vi) planejamento. Considera-se que a partir dessa pesquisa, dado a formatação das conclusões e utilização da ARA como ferramenta de trabalho, a autonomação pode ser vista e estudada através de uma estrutura lógica e dinâmica. A sua progressiva utilização por outros pesquisadores ou profissionais da área, tende a contribuir para a evolução e aprofundamento dos conceitos da autonomação, assim como, tornar os resultados da ARA mais consistentes.

A ARA a que se chegou pode e deve ser reformulada: é um instrumento vivo e que permite revisões permanentes. Aqui, ela representa o ponto de partida de um trabalho de melhoria contínua. Cabem mais estudos sobre a autonomação e seus impactos, e até mesmo, aplicações práticas em diversas indústrias. É importante salientar a viabilidade econômica de implementações voltadas para a autonomação, um assunto não tratado nesse artigo, mas, sugerido para próximos trabalhos. 


\title{
Agradecimentos
}

Parte deste trabalho foi financiado pelo CNPq.

\begin{abstract}
This paper aims at mapping the impacts of autonomation on a productive system and present, supported by Current Reality Tree (CRT), the cause-effect relationships observed in the studied procedures The paper is structured on theoretical reviews on concepts and purposes of autonomation and the Thinking Processes of the Theory of Constraints. It follows a empirical research led in a electronics parts manufacture, in which we report and set relations between entities present in a situation of interest, the manufacture routine. With a CRT, findings were related and organized in a visual fashion. Due to the tool, the findings of the research can be used as a basis to further research in the same issue.
\end{abstract}

Key-words: . Autonomation; Jidoka; Thinking Process; Theory of Constraints

\section{Referências}

ALVAREZ, R.R. Desenvolvimento de uma análise comparativa de métodos de identificação, análise e solução de problemas. 196 f. Dissertação (Mestrado em Engenharia de Produção) - Programa de Pós-graduação em Engenharia de produção, Universidade Federal do Rio Grande do Sul - UFRGS, Porto Alegre, 1996.

ANTUNES JR., J.; ALVAREZ, R.; KLIPPEL, M.; BORTOLOTTO, P. e PELLEGRIN, I. Sistemas de produção: conceitos e práticas para projeto e gestão da produção enxuta. Porto Alegre: Bookman, 2008.

ANTUNES JR., J.; KLIPPEL, M.; KOETZ, A.L. e LACERDA, D.P. Critical issues about the Theory of Constraints Thinking Process - a theoretical and practical aproach. Proceedings of the 2nd World Conference on POM and the 15th Annual POM Conference, Cancun, Mexico, 2004, p.1-11.

BLACKSTONE, J.H. Theory of constraints - a status report. International Journal of Production Research, v.39, n.6, p.1053-1080, 2001.

cross ${ }^{\text {ref }}$

BOYD, L; GUPTA, M e SUSSMAN, L. A new approach to strategy formulation: opening the black box. Journal of Education for Business, v.76, n.6, p.338-344, 2001.

cross ${ }^{\text {ref }}$

COONEY, R. Is "lean" a universal production system? Batch production in the automotive industry. International Journal of Operations \& Production Management, v.22, n.10, p.1130-1147, 2002.

cross ${ }^{\text {ref }}$

COX, J.F e SPENCER, M.S. The constraints management handbook. Boca Raton: St. Lucie Press/APICS Series on Constraints Management, 1998.

DEVARAJ, S.; HOLLINGWORTH, D.G. e SCHROEDER, R.G. Generic manufacturing strategies and plant performance. Journal of Operations Management, v.22, p.313-333, 2004.

cross ${ }^{\text {ref }}$

DOGGETT, A.M. Root cause analysis: a framework for tool selection. Quality Management Journal, v.12, n.4, p.3445, 2005.

GHINATO, P. Sistema Toyota de Produção: mais do que simplesmente just-in-time. Caxias do Sul: EDUCS, 1996.

GOLDRATT, E.M. Não é sorte: a aplicação dos processos de raciocínio da teoria das restrições. São Paulo: Nobel, 2004. 
GUPTA, M.; BOYD, L. e SUSSMAN, L. To better maps: a TOC primer for strategic planning. Business Horizons, v.47, n.2, p.15-26, 2004.

cross ${ }^{\text {ref }}$

INMAN, R.A; SALE, M.L e GREEN JR, K.W. Analysis of the relationships among TOC use, TOC outcomes, and organizational performance. International Journal of Operations \& Production Management, v.29, n.4, p.341-356, 2009.

cross ${ }^{\text {ref }}$

KIM, S; MABIN, V.J e DAVIES, J. The theory of constraints thinking processes: retrospect and prospect. International Journal of Operations \& Production Management, v.28, n.2, p.155-184, 2008.

cross ${ }^{\text {ref }}$

KLEIN, D.J e DEBRUINE, M. A thinking process for establishing management policies. Review of Business, v.16, n.3, p.31-37, 1995 .

LIKER, J.K. O modelo Toyota: 14 princípios de gestão do maior fabricante do mundo. Porto Alegre: Bookman, 2005 .

MABIN, V.J. e BALDERSTONE, S.J. The performance of the theory of constraints methodology - analysis and discussion of successful TOC applications. International Journal of Operations \& Production Management, v.23, n.6, p.568-595, 2003.

cross ${ }^{\text {ref }}$

MEHRA, S.; INMAN, R.A. e TUITE, G. A simulation-based comparison of TOC and traditional accounting performance measures in a process industry. Journal of Manufacturing Technology Management, v.16, n.3, p.328$342,2005$.

cross ${ }^{\text {ref }}$

MONDEN, Y. Sistema Toyota de Produção. São Paulo: IMAM, 1984.

OHNO, T. O Sistema Toyota de Produção: além da produção em larga escala. Porto Alegre: Bookman, 1997.

PAIVA, E.L.; CARVALHO, J.M. e FENSTERSEIFER, J.E. Estratégia de produção e operações. Porto Alegre: Bookman, 2004.

PASSOS JR., A.A. Os circuitos da autonomação - uma abordagem técnico-econômica. 96 f. Dissertação (Mestrado em Administração) - Programa de Pós-graduação em Administração, Universidade do Vale do Rio dos Sinos UNISINOS, São Leopoldo, 2004.

RAHMAN, S. The theory of constraint's thinking process approach to developing strategies in supply chains. International Journal of Physical Distribution \& Management, v.32, n.10, p.809-828, 2002.

RIBEIRO, J. e FOGLIATTO, F. Manutenção e Confiabilidade. R. Janeiro: Campus Elsevier, 2009.

RODRIGUES, L.H. Apresentação e análise crítica da Tecnologia da Produção Otimizada (Optimized Production Technology - OPT) e da Teoria das Restrições (Theory Of Constraints - TOC). Anais do XIV ENANPAD. Florianópolis: ANPAD, 1990.

SHEINKOPF, L. Thinking for a change: putting the TOC thinking process to use. Boca Raton: St. Lucie Press/APICS Series on Constraints Management, 1999.

SHINGO, S. O Sistema Toyota de Produção: do ponto de vista da engenharia de produção. Porto Alegre: Bookman, 1996.

SHINGO, S. Zero quality control: source inspection and the poka-yoke system. Portland: Productivity Press, 1986.

WATSON, K.J.; BLACKSTONE, J.H. e GARDINER, S.C. The evolution of a management philosophy: the theory of constraints. Journal of Operations Management, v.25, p.387-402, 2007.

cross' 
WOMACK, J.P.; JONES, D.T. e ROOS, D. A máquina que mudou o mundo. Rio de Janeiro: Campus, 1992.

ZILBOVICIUS, M. Modelos para a produção, produção de modelos; gênese, lógica e difusão do modelo japonês de organização da produção. São Paulo: FAPESP: Annablume, 1999.

\section{Dados dos autores:}

Nome completo: Macáliston Gonçalves da Silva

Filiação institucional: UNISINOS

Departamento: Programa de Pós-Graduação em Engenharia de Produção e Sistemas

Função ou cargo ocupado: Mestrando

Endereço completo para correspondência: Av. Unisinos, 950, São Leopoldo, RS, 93020-000

Telefones para contato: 51 3591-1122

e-mail: macaliston@ig.com.br

Nome completo: Miguel Afonso Sellitto

Filiação institucional: UNISINOS

Departamento: Programa de Pós-Graduação em Engenharia de Produção e Sistemas

Função ou cargo ocupado: Professor e pesquisador

Endereço completo para correspondência: Av. Unisinos, 950, São Leopoldo, RS, 93020-000

Telefones para contato: 51 3591-1122

e-mail: sellitto@unisinos.br 